\title{
Inclusion of Retardation Effects in the Nonrelativistic Treatment of the Lamb Shift.
}

\author{
J. SEKE and W. MöDRITSCH
}

Institut für Theoretische Physik, Technische Universität Wien

Wiedner Hauptstrasse 8-10/136, A-1040 Wien, Austria

(Nuovo Cimento D, 14, 1217 (1992))

PACS 32.90 - Other topics in atomic spectra and interactions of atoms with photons.

PACS 42.50 - Quantum optics.

PACS 3130.Jv - Radiative and relativistic effects.

PACS 99.10 - Errata.

The following typing errors should be noticed:

Equation (2.9) should read

$$
h_{1} \equiv e_{k, \lambda} \cdot \boldsymbol{P}, \quad h_{2} \equiv \frac{\boldsymbol{\sigma}}{2} \cdot\left(\hbar \boldsymbol{k} \times e_{\boldsymbol{k}, \lambda}\right),
$$

and the overall sign in eq. (3.51) should be a plus.

Furthemore, a re-examination of the numerical calculations has shown that the third term in eq. (3.56) should read

$$
M_{21 m, 3}^{(0)}=-0.04356 .
$$

This leads to the following changes in the final results for the $2 p$-state Lamb shift and the corresponding transition frequency.

The third line in eq. (3.74) should read:

$$
\Delta E_{21 m}^{\mathrm{ren}}(\boldsymbol{A} \cdot \boldsymbol{P}) / h=4.1 \mathrm{MHz} .
$$

Moreover, the value -4.14 in the $2 p$-state column of table I should be replaced two times by +4.1 .

Finally, the value 1044.8 in the column for the transition frequency $\Delta v(2 s \rightarrow 2 p)$ of table II should be replaced by 1036.5 . 